\title{
Effect of large magnetic islands on screening of external magnetic perturbation fields at slow plasma flow
}

\author{
L. Li, ${ }^{1, a)}$ Y. Q. Liu, ${ }^{2,3,4}$ X. Huang, ${ }^{1, b)}$ Q. Luan, ${ }^{5}$ and F. C. Zhong ${ }^{1}$ \\ ${ }^{1}$ College of Science, Donghua University, Shanghai 201620, China \\ ${ }^{2}$ CCFE, Culham Science Centre, Abingdon OX14 3DB, United Kingdom \\ ${ }^{3}$ Southwestern Institute of Physics, PO Box 432, Chengdu 610041, China \\ ${ }^{4}$ Department of Earth and Space Science, Chalmers University of Technology, SE-41296 Gothenburg, Sweden \\ ${ }^{5}$ School of Mathematical and Physical Sciences, Dalian University of Technology, Panjin 124221, China
}

(Received 15 November 2016; accepted 7 February 2017; published online 21 February 2017)

A toroidal resistive magneto-hydrodynamic plasma response model, involving large magnetic islands, is proposed and numerically investigated, based on local flattening of the equilibrium pressure profile near a rational surface. It is assumed that such islands can be generated near the edge of the tokamak plasma, due to the penetration of the resonant magnetic perturbations, used for the purpose of controlling the edge localized mode. Within this model, it is found that the local flattening of the equilibrium pressure helps to mitigate the toroidal curvature induced screening effect [Glasser et al., Phys. Fluids 7, 875 (1975)] — the so called Glasser-Greene-Johnson screening, when the local toroidal flow near the mode rational surface is very slow (for example, as a result of mode locking associated with the field penetration). The saturation level of the plasma response amplitude is computed, as the plasma rotation frequency approaches zero. The local modification of the plasma resistivity inside the magnetic island is found to also affect the saturation level of the plasma response at vanishing flow. Published by AIP Publishing.

[http://dx.doi.org/10.1063/1.4976987]

The type-I edge localized modes (ELMs), with low bursting frequency and large amplitude, can transiently load a large amount of heat from the plasma onto the plasma facing components within each ELM crash, which can reduce the lifetime of these components, especially for ITER. ${ }^{1}$ Recent experiments in several tokamak devices have successfully achieved the mitigation/suppression of the type-I ELMs by applying resonant magnetic perturbation (RMP) fields. $^{2-7}$ During the process of the RMP penetration, the radial transport of particles and thermal energy can be enhanced near the edge of the plasma, in particular, near the pedestal top. One channel of enhancing the transport is the presence of magnetic islands, and possibly their overlapping. In fact, recently there seems to be a solid experimental evidence from DIII-D ${ }^{8}$ and EAST, ${ }^{9}$ showing the presence of magnetic islands near the pedestal top during the ELM suppression. Large magnetic islands can partially or fully flatten the pressure profile near the resonant surface associated with the perturbation, ${ }^{10}$ which in turn can modify the field screening physics by the plasma.

The screening of the RMP fields, due to the plasma response, plays an important role in the physics understanding of ELM mitigation/suppression, ${ }^{11-16}$ especially with a slow plasma flow, which is often the case near the plasma edge. On the other hand, according to the single fluid theory, the favorable averaged toroidal curvature effect, ${ }^{17}$ at slow plasma flow, can provide a field screening effect - the so called GGJ (Glasser-Greene-Johnson) screening effect. ${ }^{18,19}$ The GGJ screening is rather different from the conventional resistive

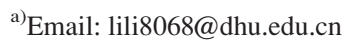

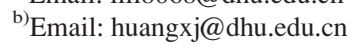

inertial (RI) screening. ${ }^{20}$ In the GGJ regime, the amplitude of the total plasma response field (the sum of the vacuum field and the field produced by the perturbed plasma current) is reduced, i.e., the screening effect is enhanced, with decreasing flow speed, ${ }^{18,19}$ whilst in the RI-regime, the response amplitude is reduced by increasing the flow speed. The GGJ screening effect has so far been investigated in the thin-island approximation. No work has been done, studying how the presence of large islands can affect the GGJ screening at a slow plasma flow. Note that the large island here does not necessarily mean the geometrical size of the island, but rather the fact that the equilibrium pressure is significantly perturbed by the presence of the island-normally either partially or fully flattened near the local rational surface.

In this work, we shall investigate the aforementioned issue, utilizing the MARS-F code ${ }^{21}$ to compute the plasma response to the external magnetic perturbation fields. MARS-F solves the single fluid, linearized resistive magneto-hydrodynamic (MHD) equations in the plasma region, together with vacuum field equations outside the plasma. For the plasma response computation, a steady state solution, in the presence of a current source located in the vacuum region, is obtained by solving a time-independent driving problem. The source current, of $\exp ($ in $\phi)$-dependence along the toroidal angle $\phi$, is specified by an array of window frame coils located near the outboard mid-plane of the torus in this study. The exact geometry of these coils, as well as the toroidal coupling among various resulting poloidal harmonics, is not an essential aspect of the present study.

In order to facilitate understanding of the key physics from the numerical results, we shall consider a simple toroidal equilibria with (near) a circular cross-section, aspect ratio 
of 10, and with a parabolic equilibrium current profile (Wesson type). The radial profiles for the equilibrium pressure and safety factor are plotted in Fig. 1. The equilibria are chosen such that there is a single rational surface (i.e., one island chain) inside the plasma at the $q=2$ surface, associated with the $n=1$ perturbation. We assume two selfconsistent equilibria, with and without the locally flattened pressure profile, that are otherwise nearly identical. Figure 1 compares the radial equilibrium pressure and the safety factor profiles between these two equilibria. It is evident that the difference in the safety factor is very minor. Note that we are comparing two extreme cases in this work-full flattening versus no-flattening. It is expected that, with a partial flattening of the pressure profile, the plasma response should be somewhere between what we find in this study.

We compute the plasma response to the external magnetic perturbation fields, as we gradually vary the plasma flow amplitude. Again for simplicity, a uniform flow profile is assumed in this study. The key numerical results are
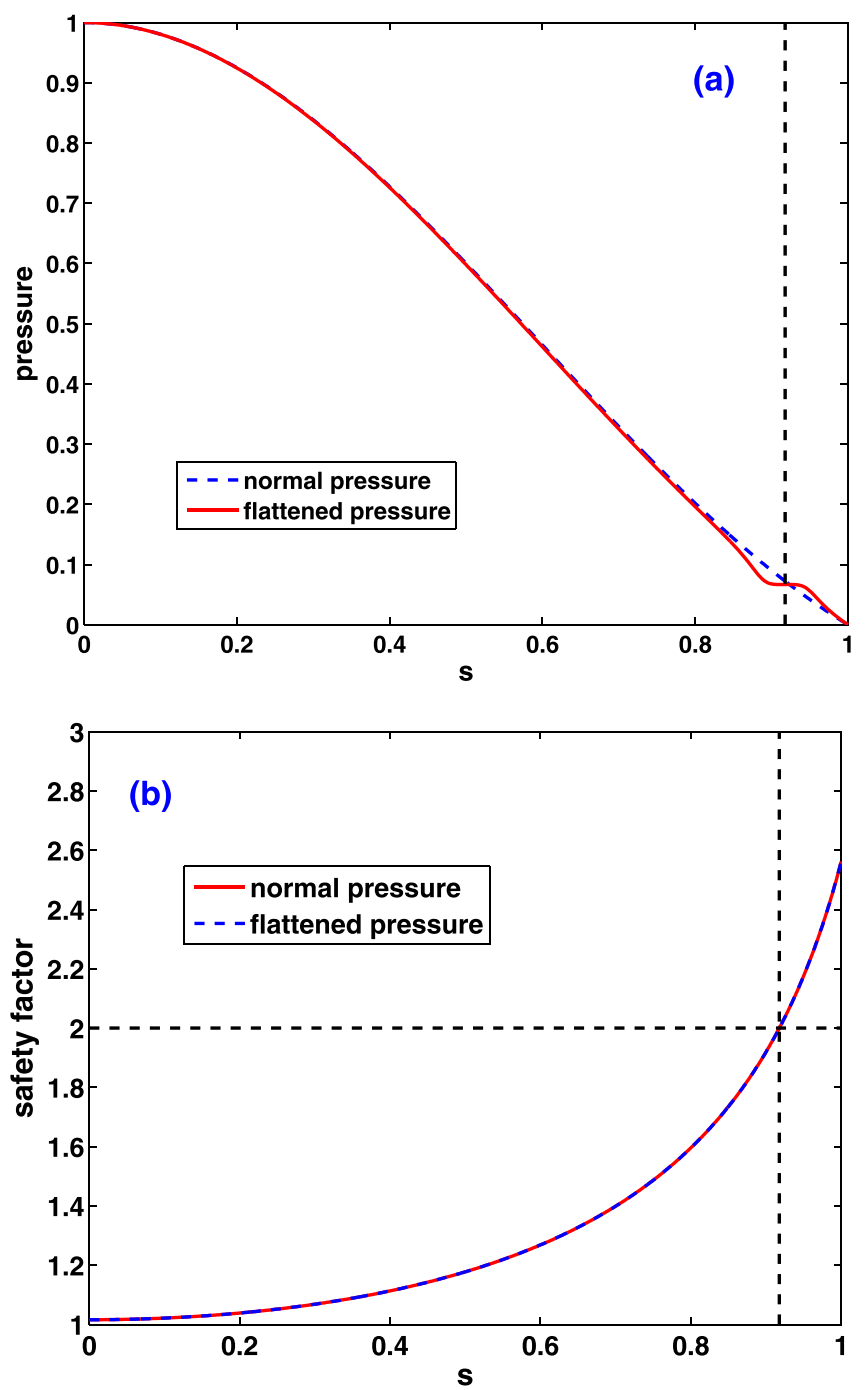

FIG. 1. Comparison of the two (with and without locally flattened pressure inside the island) radial profiles for (a) the plasma equilibrium pressure normalized by $B_{0}^{2} / \mu_{0}$ and (b) the safety factor. The radial coordinate is defined as the square root of the normalized equilibrium poloidal flux. The vertical dashed line indicates the location of the $q=2$ rational surface. summarized in Fig. 2, where we plot the amplitude of the total plasma response field, at the $q=2$ rational surface, for the $m / n=2 / 1$ harmonic of the radial field component $b_{t o t}^{1}$, normalized by the corresponding vacuum field $b_{v a c}^{1}$, versus the toroidal rotation frequency $\Omega_{q=2}$. Here, the normalized plasma resistivity is assumed to be $\eta=10^{-9}$, corresponding to the Lundquist number of $S=10^{9}$. In other words, the plasma resistivity here is normalized in such a way, that $\eta$ is the ratio of the toroidal Alfven time to the plasma resistive decay time.

It is interesting to note that, for the case with a normal pressure profile (dashed line with circles), the GGJ regime (at slow flow) and the RI regime (at fast flow) are obviously separated, as has previously been discussed. ${ }^{18,19}$ Physically, the GGJ-screening occurs when the average curvature effect becomes dominant. Since this effect is inversely proportional to the power of $3 / 2$ of the perturbation frequency in the plasma frame (in our case the perturbation is static in the laboratory frame, and thus possesses a frequency equal to the plasma toroidal rotation frequency in the plasma frame), ${ }^{17}$ the GGJ effect, which occurs due to eventual coupling of sound waves, becomes significant at a slow plasma flow. The transition from the GGJ-regime to the RI-regime occurs at the flow frequency of about $10^{-4}$ in our example. For typical tokamak plasma equilibria, this transition frequency scales as $S^{-1 / 3}$ $\left|D_{R}\right|^{2 / 3}$, ${ }^{18}$ which $D_{R}$ being the resistive interchange index.

However, with the locally flattened pressure profile near the $q=2$ surface (solid line with diamonds), the GGJ screening effect is completely removed. In other words, in the presence of a large magnetic island, the amplitude of the plasma response field approaches a constant with decreasing the plasma flow speed, meaning that, if a full RMP penetration occurs, the GGJ screening effect disappears due to the formation of large magnetic islands.

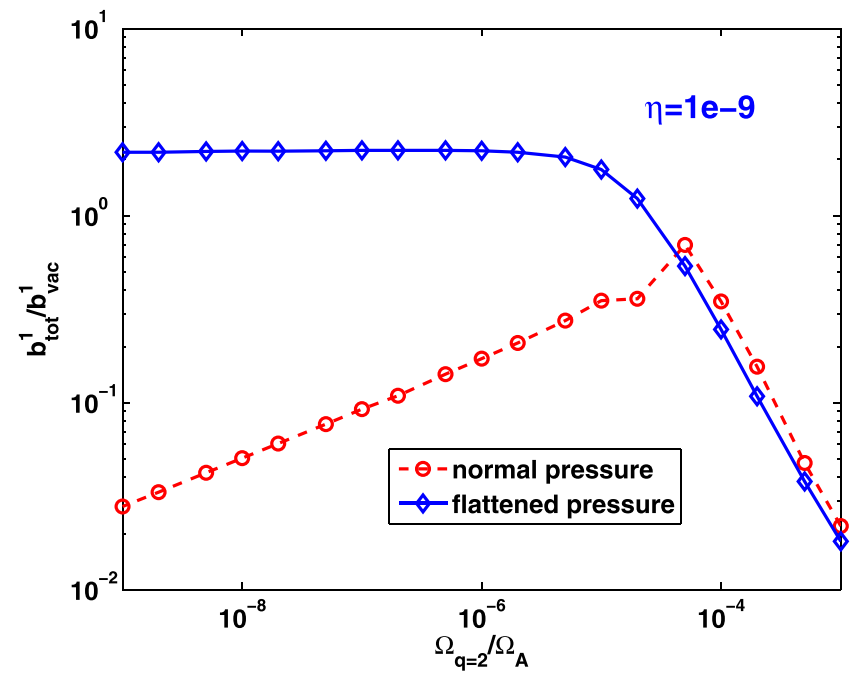

FIG. 2. The amplitude of the resonant $(m / n=2 / 1)$ radial field component, including the resistive plasma response, normalized by that of the vacuum field, versus the plasma flow speed at the rational $q=2$ surface, compared to the flow screening effects between the normal pressure profile (dashed line with circles) and the locally flattened pressure profile (solid line with diamonds). The width of the corresponding $2 / 1$ magnetic island is assumed to be $W=\Delta s=0.044$. The plasma resistivity at the $q=2$ surface corresponds to the Lundquist number of $S=10^{9}$. 
Another interesting observation is that, with the complete local flattening of the pressure profile, the saturated level of the radial magnetic field component with the plasma response, at the $q=2$ surface, approaches that of the corresponding vacuum field, as the plasma flow speed vanishes. In fact, the amplitude of the total response field is 2.19 times larger than that of the vacuum field, indicating a tearing amplification of the applied RMP field by the plasma response.

The screening effect is associated with the induced perturbed parallel current at or near the resonant surface. Such a current is generated as a result of the plasma response to the external magnetic field perturbations. Figure 3 compares the MARS-F computed perturbed parallel currents, for the two equilibria with and without local flattening of the equilibrium pressure profile. For each equilibrium, we choose two plasma rotation speeds-one from the RI regime and the other from the GGJ regime. In the RI screening regime (Figs. 3(a) and 3(c)), with $\Omega_{q=2}=10^{-4} \Omega_{A}$, the perturbed parallel current peaks near the $q=2$ surface for both equilibria, as expected. This local current sheet provides the conventional screening effect, similar to that shown in the previous work. ${ }^{19}$ On the other hand, in the slow flow regime, taking $\Omega_{q=2}=10^{-7} \Omega_{A}$ as an example here, the parallel current distribution is rather different. With the normal equilibrium pressure profile, two current peaks, being off the $q=2$ surface, are formed as shown in Fig. 3(b). This double-sheet current structure, together with a finite current density at the rational surface, eventually provides the GGJ screening effect. For the equilibrium with fully flattened pressure profile near the $q=2$ surface, the peaks of the parallel current are still (slightly) off the rational surface, but with nearly exact cancellation between the two sides. Moreover, nearly vanishing current is formed at the rational surface. These all lead to a minimal screening of the radial field component.

Inside a large magnetic island, it is expected that the plasma resistivity can also be locally modified (enhanced) due to the change of the transport process. $^{22}$ In order to investigate this effect, we assume a radially varying plasma resistivity profile, with a Gaussian function inside the $q=2$ surface
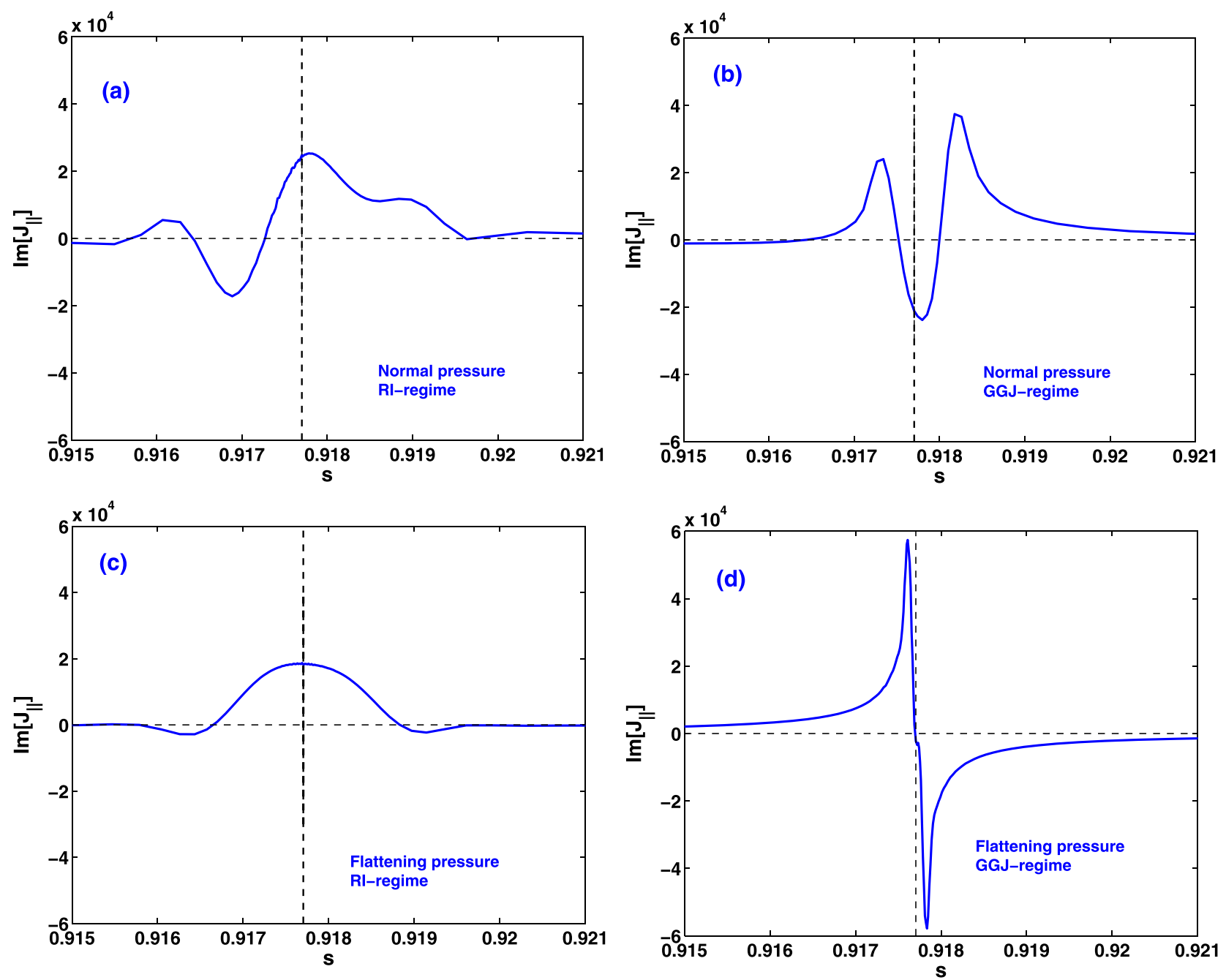

FIG. 3. Comparison of the (dominant) imaginary parts of the plasma generated, $m / n=2 / 1$ resonant parallel current density near the $q=2$ rational surface indicated by the vertical dashed lines, between the normal pressure profile ((a) $\Omega_{q=2}=10^{-4} \Omega_{A}$, (b) $\Omega_{q=2}=10^{-7} \Omega_{A}$, top panels) and the flattened pressure profile ((c) $\Omega_{q=2}=10^{-4} \Omega_{A}$, (d) $\Omega_{q=2}=10^{-7} \Omega_{A}$, bottom panels). The two rotation frequencies are associated with the RI-regime (left panel) and the GGJ-regime (right panel) respectively. The plasma resistivity at the $q=2$ surface corresponds to the Lundquist number of $S=10^{9}$. 


$$
\eta=\eta_{\text {old }}+\eta_{0} \exp \left[-\frac{\left(q-q_{\text {res }}\right)^{2}}{2 D}\right]
$$

where $\eta_{\text {old }}$ is the "background" resistivity of the plasma outside the magnetic island, $\eta_{0}$ and $D$ are numerical coefficients controlling the height and width of the resistivity profile inside the island. The plasma resistivity is normalized such that $\eta$ is the inverse of the Lundquist number $S$. We show one example in Fig. 4, with $\eta_{\text {old }}=10^{-9}, \eta_{0}=10^{-7}$, and $D=5 \times 10^{-3}$. Here plotted are both the equilibrium pressure profile, locally flattened near the rational surface, and the plasma resistivity profile with a Gaussian function inside the flattened pressure region. Note that we only plot the shape of the profiles, with the peak amplitude being normalized to unity for both profiles.

We now scan the amplitude of the local plasma resistivity (at the $q=2$ surface), assuming the same equilibrium with flattened pressure. The computed plasma response field amplitude is plotted in Fig. 5. Since the plasma response at a slow plasma rotation regime is associated with the RMP penetration, here we consider a very slow plasma flow case, with $\Omega_{0}=10^{-7} \Omega_{A}$. The resistivity outside the island is still assumed to be $\eta_{\text {old }}=10^{-9}$. The plasma response amplitude saturates with increasing the plasma resistivity inside the magnetic island. The saturated level is still close but slightly higher than that of the vacuum field. This may be associated with the resonant field amplification effect introduced by the plasma response. At the other end of $\eta_{0}\left(\eta_{0}=10^{-9}\right)$, the plasma response nearly recovers the value shown in Fig. 2. The slight difference comes from the finite (Gaussian) correction of the plasma resistivity (inside the island) to the background resistivity $\left(\eta_{\text {old }}\right)$, with the latter being assumed to be the sole component in producing Fig. 2.

The resonant field amplification effect, reported in Figs. 5 and 2 (for the case with flattened pressure profile at slow plasma flow), is due to combined effects from several physics, including the local and global equilibrium current and pressure profiles, the local plasma resistivity and flow

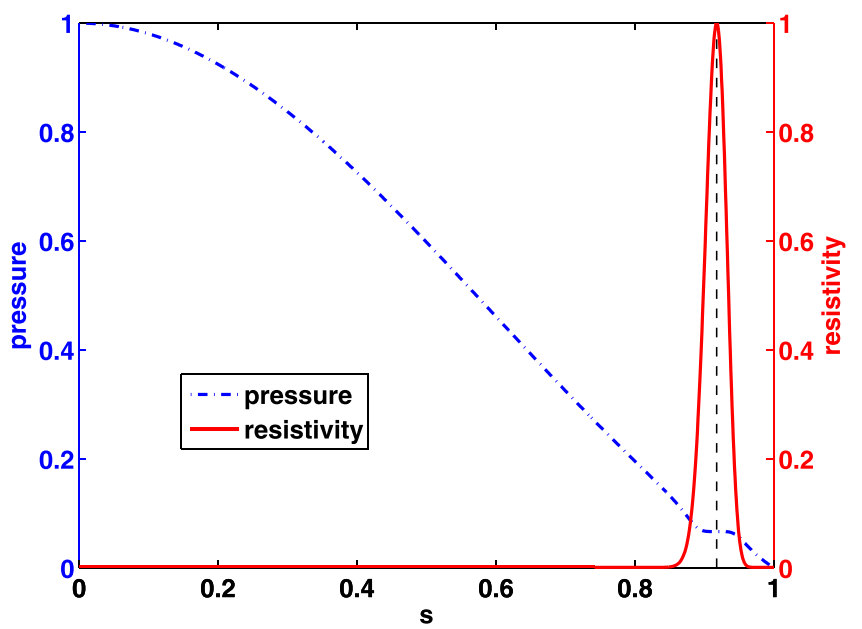

FIG. 4. A typical shape of the radial profile for the plasma resistivity (normalized to unity for the peak value), artificially enhanced inside the magnetic island, plotted together with the shape of the plasma equilibrium pressure profile (normalized to unity at the magnetic axis), locally flattened near the $q=2$ rational surface.

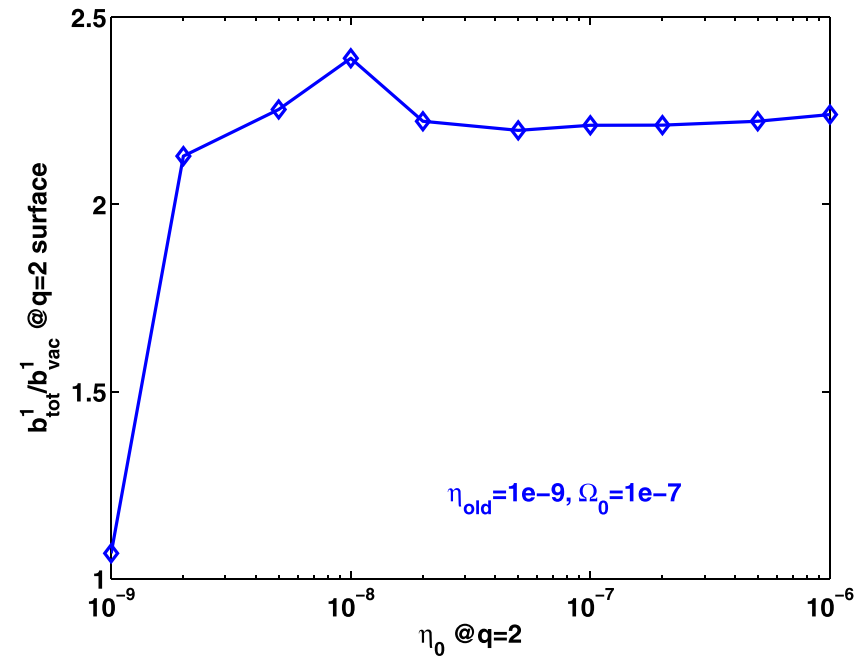

FIG. 5. The amplitude of the resonant $(m / n=2 / 1)$ radial field component including the resistive plasma response, normalized by that of the vacuum field, versus the parameter $\eta_{0}$ as defined in Eq. (1). The normalized plasma resistivity outside the island is assumed to be $\eta_{\text {old }}=10^{-9}$, corresponding to the Lundquist number of $S=10^{9}$. The plasma flow is fixed at $\Omega_{0}=10^{-7} \Omega_{A}$, corresponding to the GGJ regime. Considered is an equilibrium with a flattened pressure profile near the $q=2$ rational surface.

speed near the rational surface, as well as the geometrical coupling factors. Therefore, this type of amplification does not always occur in either of the two regimes considered in this work.

In summary, understanding the RMP penetration physics is important for the suppression of the ELMs. By assuming the presence of a large magnetic island near the plasma edge, we investigate an equilibrium model where the pressure profile is locally flattened near a rational surface. The numerical results with MARS-F show that the GGJ screening effect can be completely removed by large islands. Such a loss of the field screening is associated with substantial modification of the radial structure in the plasma generated shielding current. The amplitude of the plasma response inside the resonant layer approaches a value, which is close to but larger than that of the corresponding vacuum field. Taken into account, the possible modification of the plasma resistivity inside a large island, the plasma response amplitude is found to saturate at sufficiently a large plasma resistivity. These numerical findings may provide a crucial understanding of the ELMs suppression physics. In this work, a simple equilibrium model, including a single rational surface inside the plasma, is considered. More realistic toroidal equilibria will be considered in the future work.

Finally, we discuss some other physics that may be relevant to our study here and hence affecting the results presented in this work. These physics models have been briefly discussed in a recent review. ${ }^{23}$ The first is the two fluid effect, which has been analytically thoroughly investigated in Refs. 14 and 24, in terms of the layer response and the resonant field screening. No field line curvature effect, nor the sound wave coupling (parallel compressibility), however, has been taken into account in these two-fluid models. It should be interesting to develop a two-fluid layer theory that does include these effects, which are the essential ingredients 
of the GGJ effect. In the presence of a large magnetic island, the electron diamagnetic flow inside the layer may be reduced or even completely removed, due to the pressure flattening. Consequently, the relevant flow, responsible for the eventual screening of the field, can well be the $E \times B$ flow instead of the electron flow, as has recently been pointed out. ${ }^{25}$ The next interesting physics is the neoclassical effect, in particular, the role of (missing) bootstrap current in the presence of large magnetic islands. This physics is important for the neoclassical tearing mode (NTM), ${ }^{26}$ and can certainly be important also for the plasma response in the presence of a finite size island. In the NTM theory, the key role is played by the perturbed bootstrap current associated with the helical pressure perturbation. In this work, we assume that the equilibrium pressure is locally modified only by the axisymmetric $(n=0)$ perturbation. Generally speaking, the effect of non-axisymmetric pressure perturbation can only be properly studied using three-dimensional equilibrium model, which is beyond the current capability of the MARS-F code. On the other hand, the anisotropic (parallel versus perpendicular) thermal conduction model, also incorporated into the NTM theory ${ }^{26}$ can be utilized in combination with the standard single fluid model. The anisotropic thermal conduction directly applies to the helical pressure perturbation, thus eliminating the necessity for the ad-hoc assumption of the local pressure flattening. The model has recently been implemented into the MARS-F code, and a similar study of the GGJ screening, in the presence of thermal conduction, is currently ongoing. The third important effect, that is missing from the present study, is the kinetic effect. It is well known that a full kinetic treatment of the resistive layer is a complicated problem, involving multiple spatial and time scales for ions and electrons. Certain aspects of the drift kinetic effects, however, can be addressed by the self-consistent MHDkinetic hybrid model incorporated into the MARS-K code. ${ }^{27}$ This remains the future work. Finally, the ad-hoc approach that we take in this study, is probably reasonable in studying the final stage of the RMP field penetration (as well as in comparison with the initial, fully shielded stage). The full dynamic process of the penetration, e.g., the dynamic formation of large islands, the resulting local pressure flattening, the change of the plasma rotation, certainly calls for non-linear time evolution simulations. Certain aspects of the quasi-linear time evolution though, associated with the GGJ regime, can be studied using the MARS-Q model. ${ }^{28}$ This will be investigated in the near future.

This work was supported by the Fund of National Natural Science Foundation of China (NSFC) [Grant Nos. 11405029, 11275047, 11505021, 11305035, and 11428512], the National Magnetic Confinement Fusion Science Program under Grant No. 2015GB104004, and the Fund of DUT15RC(3)039.

\footnotetext{
${ }^{1}$ A. Loarte, G. Huijsmans, S. Futatani, L. R. Baylor, T. E. Evans, D. M. Orlov, O. Schmitz, M. Becoulet, P. Cahyna, Y. Gribov, A. Kavin, A. Sashala Naik, D. J. Campbell, T. Casper, E. Daly, H. Frerichs, A. Kischner, R. Laengner, S. Lisgo, R. A. Pitts, G. Saibene, and A. Wingen, Nucl. Fusion 54, 33007 (2014).
}

${ }^{2}$ T. E. Evans, R. A. Moyer, P. R. Thomas, J. G. Watkins, T. H. Osborne, J. A. Boedo, E. J. Doyle, M. E. Fenstermacher, K. H. Finken, R. J. Groebner, M. Groth, J. H. Harris, R. J. La Haye, C. J. Lasnier, S. Masuzaki, N. Ohyabu, D. G. Pretty, T. L. Rhodes, H. Reimerdes, D. L. Rudakov, M. J. Schaffer, G. Wang, and L. Zeng, Phys. Rev. Lett. 92, 235003 (2004).

${ }^{3}$ R. J. Hawryluk, D. J. Campbell, G. Janeschitz, P. R. Thomas, R. Albanese, R. Ambrosino, C. Bachmann, L. Baylor, M. Becoulet, I. Benfatto, J. Bialek, A. Boozer, A. Brooks, R. Budny, T. Casper, M. Cavinato, J. J. Cordier, V. Chuyanov, E. Doyle, T. Evans, G. Federici, M. Fenstermacher, H. Fujieda, K. G'al, A. Garofalo, L. Garzotti, D. Gates, Y. Gribov, P. Heitzenroeder, T. C. Hender, N. Holtkamp, D. Humphreys, I. Hutchinson, K. Ioki, J. Johner, G. Johnson, Y. Kamada, A. Kavin, C. Kessel, R. Khayrutdinov, G. Kramer, A. Kukushkin, K. Lackner, I. Landman, P. Lang, Y. Liang, J. Linke, B. Lipschultz, A. Loarte, G. D. Loesser, C. Lowry, T. Luce, V. Lukash, S. Maruyama, M. Mattei, J. Menard, M. Merola, A. Mineev, N. Mitchel, E. Nardon, R. Nazikian, B. Nelson, C. Neumeyer, J.-K. Park, R. Pearce, R. A. Pitts, A. Polevoi, A. Portone, M. Okabayashi, P. H. Rebut, V. Riccardo, J. Roth, S. Sabbagh, G. Saibene, G. Sannazzaro, M. Schaffer, M. Shimada, A. Sen, A. Sips, C. H. Skinner, P. Snyder, R. Stambaugh, E. Strait, M. Sugihara, E. Tsitrone, J. Urano, M. Valovic, M. Wade, J. Wesley, R. White, D. G. Whyte, S. Wu, M. Wykes, and L. Zakharov, Nucl. Fusion 49, 065012 (2009).

${ }^{4}$ Y. Liang, H. R. Koslowski, P. R. Thomas, E. Nardon, B. Alper, P. Andrew, Y. Andrew, G. Arnoux, Y. Baranov, M. Bécoulet, M. Beurskens, T. Biewer, M. Bigi, K. Crombe, E. De La Luna, P. de Vries, W. Fundamenski, S. Gerasimov, C. Giroud, M. P. Gryaznevich, N. Hawkes, S. Hotchin, D. Howell, S. Jachmich, V. Kiptily, L. Moreira, V. Parail, S. D. Pinches, E. Rachlew, and O. Zimmermann, Phys. Rev. Lett. 98, 265004 (2007).

${ }^{5}$ A. Kirk, E. Nardon, R. Akers, M. Bécoulet, G. De Temmerman, B. Dudson, B. Hnat, Y. Q. Liu, R. Martin, P. Tamain, D. Taylor, and MAST Team, Nucl. Fusion 50, 034008 (2010).

${ }^{6}$ W. Suttrop, T. Eich, J. C. Fuchs, S. Günter, A. Janzer, A. Herrmann, A. Kallenbach, P. T. Lang, T. Lunt, M. Maraschek, R. M. McDermott, A. Mlynek, T. Pütterich, M. Rott, T. Vierle, E. Wolfrum, Q. Yu, I. Zammuto, and H. Zohm (ASDEX Upgrade Team), Phys. Rev. Lett. 106, 225004 (2011).

${ }^{7}$ Y. M. Jeon, J.-K. Park, S. W. Yoon, W. H. Ko, S. G. Lee, K. D. Lee, G. S. Yun, Y. U. Nam, W. C. Kim, J.-G. Kwak, K. S. Lee, H. K. Kim, and H. L. Yang (KSTAR Team), Phys. Rev. Lett. 109, 035004 (2012).

${ }^{8}$ R. Nazikian, C. Paz-Soldan, J. D. Callen, J. S. deGrassie, D. Eldon, T. E. Evans, N. M. Ferraro, B. A. Grierson, R. J. Groebner, S. R. Haskey, C. C. Hegna, J. D. King, N. C. Logan, G. R. McKee, R. A. Moyer, M. Okabayashi, D. M. Orlov, T. H. Osborne, J.-K. Park, T. L. Rhodes, M. W. Shafer, P. B. Snyder, W. M. Solomon, E. J. Strait, and M. R. Wade, Phys. Rev. Lett. 114, 105002 (2015).

${ }^{9}$ Y. Sun, Y. Liang, Y. Q. Liu, S. Gu, X. Yang, W. Guo, T. Shi, M. Jia, L. Wang, B. Lyu, C. Zhou, A. Liu, Q. Zang, H. Liu, N. Chu, H. H. Wang, T. Zhang, J. Qian, L. Xu, K. He, D. Chen, B. Shen, X. Gong, X. Ji, S. Wang, M. Qi, Y. Song, Q. Yuan, Z. Sheng, G. Gao, P. Fu, and B. Wan, Phys. Rev. Lett. 117, 115001 (2016).

${ }^{10}$ B. D. Scott, A. B. Hassam, and J. F. Drake, Phys. Fluids 28, 275 (1985).

${ }^{11}$ Y. Q. Liu, A. Kirk, and E. Nardon, Phys. Plasmas 17, 122502 (2010).

${ }^{12}$ Y. Q. Liu, A. Kirk, Y. Gribov, M. P. Gryaznevich, T. C. Hender, and E. Nardon, Nucl. Fusion 51, 083002 (2011)

${ }^{13}$ F. Martin Heyn, B. Ivan Ivanov, V. Sergei Kasilov, K. W. Kernbichler, I. Joseph, A. R. Moyer, and M. A. Runov, Nucl. Fusion 48, 024005 (2008).

${ }^{14}$ F. L. Waelbroeck, I. Joseph, E. Nardon, M. Bécoulet, and R. Fitzpatrick, Nucl. Fusion 52, 074004 (2012).

${ }^{15}$ N. M. Ferraro, Phys. Plasmas 19, 056105 (2012).

${ }^{16}$ F. Orain, M. Bécoulet, G. Dif-Pradalier, G. Huijsmans, S. Pamela, E. Nardon, C. Passeron, G. Latu, V. Grandgirard, A. Fil, A. Ratnani, I. Chapman, A. Kirk, A. Thornton, M. Hoelzl, and P. Cahyna, Phys. Plasmas 20, 102510 (2013).

${ }^{17}$ A. H. Glasser, J. M. Greene, and J. L. Johnson, Phys. Fluids 18, 875 (1975).

${ }^{18}$ Y. Q. Liu, J. W. Connor, S. C. Cowley, C. J. Ham, R. J. Hastie, and T. C. Hender, Phys. Plasmas 19, 072509 (2012).

${ }^{19}$ L. Li, Y. Q. Liu, Y. Liang, N. Wang, Q. Luan, F. C. Zhong, and Y. Liu, Nucl. Fusion 56, 092008 (2016).

${ }^{20}$ R. Fitzpatrick, Nucl. Fusion 33, 1049 (1993).

${ }^{21}$ Y. Q. Liu, A. Bondeson, C. M. Fransson, B. Lennartson, and C. Breitholtz, Phys. Plasmas 7, 3681 (2000).

${ }^{22}$ W. Gekelman, R. L. Stenzel, and N. Wild, Phys. Scr. 1982, 277. 
${ }^{23}$ Y. Liu, C. J. Ham, A. Kirk, L. Li, A. Loarte, D. A. Ryan, Y. Sun, W. Suttrop, X. Yang, and L. Zhou, Plasma Phys. Controlled Fusion 58, 114005 (2016).

${ }^{24}$ A. Cole and R. Fitzpatrick, Phys. Plasmas 13, 032503 (2006).

${ }^{25}$ J. D. Callen, R. Nazikian, N. M. Ferraro, M. T. Beidler, C. C. Hegna, R. J.

La Haye, and C. Paz-Soldan, "Model of ELM suppression by RMPs in
DIII-D," in Proceedings of 58th Annual Meeting of the APS Division of Plasma Physics, abstract \#PP10.00070, Vol. 61, No. 18.

${ }^{26}$ R. Fitzpatrick, Phys. Plasmas 2, 825 (1995).

${ }^{27}$ Y. Q. Liu, M. S. Chu, I. T. Chapman, and T. C. Hender, Phys. Plasmas 15 112503 (2008).

${ }^{28}$ Y. Q. Liu, A. Kirk, and Y. Sun, Phys. Plasmas 20, 042503 (2013). 